\title{
A proof of the Grünbaum conjecture
}

by

\section{Bruce L. Chalmers (Riverside, CA) and Grzegorz Lewicki (Kraków)}

\begin{abstract}
Let $V$ be an $n$-dimensional real Banach space and let $\lambda(V)$ denote its absolute projection constant. For any $N \in \mathbb{N}$ with $N \geq n$ define

$$
\lambda_{n}^{N}=\sup \left\{\lambda(V): \operatorname{dim}(V)=n, V \subset l_{\infty}^{(N)}\right\}, \quad \lambda_{n}=\sup \{\lambda(V): \operatorname{dim}(V)=n\} .
$$

A well-known Grünbaum conjecture [Trans. Amer. Math. Soc. 95 (1960)] says that

$$
\lambda_{2}=4 / 3 \text {. }
$$

König and Tomczak-Jaegermann [J. Funct. Anal. 119 (1994)] made an attempt to prove this conjecture. Unfortunately, their Proposition 3.1, used in the proof, is incorrect. In this paper a complete proof of the Grünbaum conjecture is presented.
\end{abstract}

1. Introduction. Let $X$ be a real Banach space and let $V \subset X$ be a finite-dimensional subspace. A continuous linear mapping $P: X \rightarrow V$ is called a projection if $\left.P\right|_{V}=\left.\mathrm{id}\right|_{V}$. Denote by $\mathcal{P}(X, V)$ the set of all projections from $X$ onto $V$. Set

$$
\begin{aligned}
\lambda(V, X) & =\inf \{\|P\|: P \in \mathcal{P}(X, V)\}, \\
\lambda(V) & =\sup \{\lambda(V, X): V \subset X\} .
\end{aligned}
$$

The constant $\lambda(V, X)$ is called the relative projection constant and $\lambda(V)$ the absolute projection constant. General bounds for absolute projection constants were studied by many authors (see e.g. [1, 2, 1, 8, 9, 11, 12]). It is well-known (see e.g [13]) that if $V$ is a finite-dimensional space then

$$
\lambda(V)=\lambda\left(I(V), l_{\infty}\right\},
$$

where $I(V)$ denotes any isometric copy of $V$ in $l_{\infty}$. For any $n \in \mathbb{N}$ denote

$$
\lambda_{n}=\sup \{\lambda(V): \operatorname{dim}(V)=n\}
$$

and for any $N \in \mathbb{N}$ with $N \geq n$,

$$
\lambda_{n}^{N}=\sup \left\{\lambda(V): V \subset l_{\infty}^{(N)}\right\} .
$$

2010 Mathematics Subject Classification: Primary 46A22, 47A30; Secondary 47A58. Key words and phrases: absolute projection constant, minimal projection. 
By the Kadec-Snobar Theorem [6], $\lambda_{n} \leq \sqrt{n}$ for any $n \in \mathbb{N}$. However, determination of the constant $\lambda_{n}$ seems to be difficult. In [5, p. 465] it was conjectured by B. Grünbaum that

$$
\lambda_{2}=4 / 3 .
$$

In [10, Th. 1.1] an attempt has been made to prove this conjecture (and a more general result). The proof presented in that paper is mainly based on [10, Proposition 3.1, p. 259 and Lemma 5.1, p. 273]. Unfortunately, the proof of Proposition 3.1 is incorrect. In fact the formula (3.19) from [10, p. 263] is false. This can be easily checked by differentiating formula (3.12) on page 262 there with respect to $Z_{s 1}$ (we are using the notation of [10]). Because of this error, part of the proof of [10, Proposition 3.1 on p. 265] is incorrect and as a result, the proof of [10, Th. 1.1, p. 255] is incomplete. Moreover, not only the proof of Proposition 3.1 from [10] is incorrect but also its statement (see [3]).

In this paper we give a proof of the Grünbaum conjecture. In Section 2 we present some preliminary results used in the proof. Section 3 contains the proof of the Grünbaum conjecture. The main tools applied are the Lagrange Multiplier Theorem and the Implicit Function Theorem. Since our paper is rather technical, we describe the relations between the results contained in Sections 2 and 3.

The final part of the proof of the Grünbaum conjecture is presented in Theorem 3.2. In its proof we need Theorems 3.1 and 2.4 and Lemma 2.13 .

Theorem 3.1 is mainly a consequence of Theorems 2.21 and 2.22, which are the crucial technical results of this paper. (In our proof of Theorem 3.1. Lemmas 2.5 and 2.6 and Theorems 2.11, 2.16, 2.17 and 2.18 are also applied.).

Theorem 2.21 is based on Lemmas 2.2, 2.8, 2.15 and 2.19 and Theorems 2.16 and 2.17 .

Theorem 2.22 is mainly a consequence of Lemmas 2.9 and 2.10 .

Lemma 2.12 is applied in the proof of Theorem 2.16, Lemma 2.13 in the proof of Theorem 2.17, and Lemma 2.14 in the proof of Theorem 2.18. Also we need Theorem 2.3 in the proof of Theorem 2.11, and Lemma 2.1 in the proof of Lemma 2.15.

In the proofs of Theorems 2.11 and 2.22 and Lemmas 2.122 .14 our symbolic Mathematica programs were used. The source files for them are available at http://www2.im.uj.edu.pl/GrzegorzLewicki/GrunbaumConjecture/ (later referred to as "our web site").

2. Preliminaries. In this section we mainly consider the following problem: for a fixed $u_{1} \in[0,1]$ maximize the function $f_{u_{1}}: \mathbb{R}^{N-1} \times\left(\mathbb{R}^{N}\right)^{n} \rightarrow \mathbb{R}$ 
defined by

$$
f_{u_{1}}\left(\left(u_{2}, \ldots, u_{N}\right), x^{1}, \ldots, x^{n}\right)=\sum_{i, j=1}^{N} u_{i} u_{j}\left|\left\langle x_{i}, x_{j}\right\rangle_{n}\right|
$$

under the constraints

$$
\begin{gathered}
\left\langle x^{i}, x^{j}\right\rangle_{N}=\delta_{i j}, \quad 1 \leq i \leq j \leq n, \\
\sum_{j=2}^{N} u_{j}^{2}=1-u_{1}^{2} .
\end{gathered}
$$

Here for $j=1, \ldots, N, x_{j}=\left(\left(x^{1}\right)_{j}, \ldots,\left(x^{n}\right)_{j}\right),\langle w, z\rangle_{n}=\sum_{j=1}^{n} w_{j} z_{j}$ for any $w=\left(w_{1}, \ldots, w_{n}\right), z=\left(z_{1}, \ldots, z_{n}\right) \in \mathbb{R}^{n}$ and $\langle p, q\rangle_{N}=\sum_{j=1}^{N} p_{j} q_{j}$ for any $p=\left(p_{1}, \ldots, p_{N}\right), q=\left(q_{1}, \ldots, q_{N}\right) \in \mathbb{R}^{N}$. Also we will work with

$$
f_{u_{1}, A}\left(\left(u_{2}, \ldots, u_{N}\right), x^{1}, \ldots, x^{n}\right)=\sum_{i, j=1}^{N} u_{i} u_{j} a_{i j}\left\langle x_{i}, x_{j}\right\rangle_{n}
$$

where $A=\left\{a_{i j}\right\}$ is a fixed $N \times N$ symmetric matrix.

Now we state some results which will be of use later. Their proofs can be found in [3].

Lemma $2.1([3])$. Let $x^{1}, \ldots, x^{n} \in \mathbb{R}^{N}$ and $u \in \mathbb{R}^{N}$ satisfy 2.2, 2.3]. Set $V=\operatorname{span}\left[x^{1}, \ldots, x^{n}\right]$. Assume $v^{1}, \ldots, v^{n}$ is an orthonormal basis of $V$ (with respect to $\left.\langle\cdot, \cdot\rangle_{N}\right)$. Then

$$
f_{u_{1}}\left(\left(u_{2}, \ldots, u_{N}\right), x^{1}, \ldots, x^{n}\right)=f_{u_{1}}\left(\left(u_{2}, \ldots, u_{N}\right), v^{1}, \ldots, v^{n}\right)
$$

and

$$
f_{u_{1}, A}\left(\left(u_{2}, \ldots, u_{N}\right), x^{1}, \ldots, x^{n}\right)=f_{u_{1}, A}\left(\left(u_{2}, \ldots, u_{N}\right), v^{1}, \ldots, v^{n}\right)
$$

for any $N \times N$ symmetric matrix $A$.

Lemma $2.2([3])$. Let $n, N \in \mathbb{N}$ with $N \geq n$. Fix $u=\left(u_{1}, \ldots, u_{N}\right) \in \mathbb{R}^{N}$ with nonnegative coordinates. Let $f: \mathbb{R}^{n N} \rightarrow \mathbb{R}$ be given by

$$
f\left(x^{1}, \ldots, x^{n}\right)=\sum_{i, j=1}^{N} u_{i} u_{j}\left|\left\langle x_{i}, x_{j}\right\rangle_{n}\right|,
$$

where $x^{i} \in \mathbb{R}^{N}$ for $i=1, \ldots, n$. Assume that $y^{1}, \ldots, y^{n} \in \mathbb{R}^{N}$ are so chosen that

$$
f\left(y^{1}, \ldots, y^{n}\right)=\max \left\{f\left(x^{1}, \ldots, x^{n}\right):\left(x^{1}, \ldots, x^{n}\right) \text { satisfy } 2.2\right\} .
$$

Let $A \in \mathbb{R}^{N \times N}$ be the matrix defined by

$$
a_{i j}=\operatorname{sgn}\left(\left\langle y_{i}, y_{j}\right\rangle_{n}\right)
$$


for $i, j=1, \ldots, N(\operatorname{sgn}(0)=1$ by definition $)$. Define $B \in \mathbb{R}^{N \times N}$ by

$$
b_{i j}=u_{i} u_{j} a_{i j}
$$

for $i, j=1, \ldots, N$. Let $b_{1} \geq \cdots \geq b_{N}$ denote the eigenvalues of $B$ (since $B$ is symmetric, all of them are real). Then there exist orthonormal (with respect to $\left.\langle\cdot, \cdot\rangle_{N}\right)$ eigenvectors $w^{1}, \ldots, w^{n} \in \mathbb{R}^{N}$ of $B$ corresponding to $b_{1}, \ldots, b_{n}$ such that

$$
f\left(w^{1}, \ldots, w^{n}\right)=f\left(y^{1}, \ldots, y^{n}\right)=\sum_{j=1}^{n} b_{j} .
$$

Set

$$
f_{1}\left(x^{1}, \ldots, x^{n}\right)=\sum_{i, j=1}^{N} b_{i j}\left\langle x_{i}, x_{j}\right\rangle_{n} .
$$

If $y^{1}, \ldots, y^{n} \in \mathbb{R}^{N}$ are such that

$$
\begin{aligned}
f_{1}\left(y^{1}, \ldots, y^{n}\right) & =\max \left\{f_{1} \text { under constraint } 2.20\right\} \\
& =\max \{f \text { under constraint } 2.2]\}
\end{aligned}
$$

and $b_{n}>b_{n+1}$ then $\operatorname{span}\left[y^{i}: i=1, \ldots, n\right]=\operatorname{span}\left[w^{i}: i=1, \ldots, n\right]$.

Theorem $2.3([3])$. Let $\mathcal{A}$ denote the set of all $N \times N$ symmetric matrices $\left(a_{i j}\right)$ such that $a_{i j}= \pm 1$ and $a_{i i}=1$ for $i, j=1, \ldots, N$. Let $f_{u_{1}}$ be given by 2.1. Then

$\max \left\{f_{u_{1}}:\left(\left(u_{2}, \ldots, u_{N}\right), x^{1}, \ldots, x^{n}\right)\right.$ satisfying $\left.\left.2.2,2.3\right)\right\}$

$$
=\max \left\{\sum_{i=1}^{n} b_{i}(v, A): A \in \mathcal{A}, v=\left(v_{1}, \ldots, v_{n}\right) \in \mathbb{R}^{N}, \sum_{i=1}^{N} v_{i}^{2}=1, v_{1}=u_{1}\right\},
$$

where $b_{1}(v, A) \geq \cdots \geq b_{n}(v, A)$ denote the $n$ largest eigenvalues of the $N \times N$ matrix $\left(v_{i} v_{j} a_{i j}\right)_{i, j=1}^{N}$. Also

$$
\begin{aligned}
& \left.\max \left\{\sum_{i, j=1}^{N} u_{i} u_{j}\left|\left\langle x_{i}, x_{j}\right\rangle_{n}\right|:\left(x^{1}, \ldots, x^{n}\right) \text { satisfy } 2.2\right), \sum_{j=1}^{N} u_{j}^{2}=1\right\} \\
& \quad=\max \left\{\sum_{i=1}^{n} b_{i}(v, A): A \in \mathcal{A}, v=\left(v_{1}, \ldots, v_{n}\right) \in \mathbb{R}^{N}, \sum_{i=1}^{N} v_{i}^{2}=1\right\} .
\end{aligned}
$$

Now for $n, N \in \mathbb{N}$ with $N \geq n$ define

$$
\lambda_{n}^{N}=\sup \left\{\lambda\left(V, l_{\infty}^{(N)}\right): V \subset l_{\infty}^{(N)}, \operatorname{dim}(V)=n\right\} .
$$

Theorem $2.4([\underline{3}])$. Let $n, N \in \mathbb{N}$ with $N \geq n$. Then

$$
\lambda_{n}^{N}=\max \left\{\sum_{i, j=1}^{N} u_{i} u_{j}\left|\left\langle x_{i}, x_{j}\right\rangle_{n}\right|:\left(x^{1}, \ldots, x^{n}\right) \text { satisfy }(2.2), \sum_{j=1}^{N} u_{j}^{2}=1\right\} \text {. }
$$


Lemma 2.5 ([3]). For any $n \geq 2$,

$$
\lambda_{n}^{n+1}=2-2 /(n+1) .
$$

Moreover, $\lambda_{n}^{n+1}=\lambda\left(\operatorname{ker}(f), l_{\infty}^{(n+1)}\right)$ if and only if $f=c( \pm 1, \ldots, \pm 1)$, where $c$ is a positive constant.

Lemma 2.6 ([3]). Consider problem (2.1) with $u_{1}=0$ and fixed $N \geq n+2$. Assume that $\lambda_{n}^{N-1}>\lambda_{n-1}^{N-1}$. Then the maximum of $f_{u_{1}}$ under constraints 2.2 , 2.3 is equal to $\lambda_{n}^{N-1}$.

Lemma 2.7 ([3]). Let $u=\left(u_{1}, \ldots, u_{N}\right) \in \mathbb{R}^{N}$ and let $z=\left(z_{2}, \ldots, z_{n}\right) \in$ $\{-1,1\}^{N-1}$. Let $A_{z}$ be the $N \times N$ symmetric matrix defined by $a_{1 j}=z_{j} \in$ $\{ \pm 1\}$ for $j=2, \ldots, N, a_{i j}=-1$ for $i, j=2, \ldots, N, i \neq j$, and $a_{i i}=1$ for $i=1, \ldots, N$. Let $B_{z}=\left(u_{i} u_{j}\left(A_{z}\right)_{i j}\right)_{i, j=1}^{N}$. Hence

$$
B_{z}=\left(\begin{array}{ccccc}
u_{1}^{2} & z_{2} u_{1} u_{2} & z_{3} u_{1} u_{3} & \ldots & z_{N} u_{1} u_{N} \\
z_{2} u_{1} u_{2} & u_{2}^{2} & -u_{2} u_{3} & \ldots & -u_{2} u_{N} \\
z_{3} u_{1} u_{2} & -u_{2} u_{3} & u_{3}^{2} & \ldots & -u_{2} u_{N} \\
\ldots & \ldots & \ldots & \ldots & \ldots \\
z_{N} u_{1} u_{N} & -u_{2} u_{N} & \ldots & \ldots & u_{N}^{2}
\end{array}\right) .
$$

Let $\sigma$ be a permutation of $\{1, \ldots, N\}$ such that $\sigma(1)=1$ and for any $x=$ $\left(x_{1}, \ldots, x_{N}\right) \in \mathbb{R}^{N}$, let $x_{-}=\left(x_{1},-x_{2}, \ldots,-x_{N}\right)$. Then the matrices

$$
B_{\sigma(z)}=\left(u_{\sigma(i)} u_{\sigma(j)}(A \sigma(z))_{i j}\right)_{i, j=1}^{N}, \quad B_{z_{-}}=\left(\left(u_{i} u_{j}\left(A_{z_{-}}\right)_{i j}\right)_{i, j=1}^{N}\right.
$$

and $B_{z}$ have the same eigenvalues.

The next lemma is a simple consequence of the Implicit Function Theorem.

Lemma $2.8([3])$. Let $U \subset \mathbb{R}^{l}$ be an open nonempty set and let $f$ : $U \times \mathbb{R}^{n} \rightarrow \mathbb{R}$ and $G_{i}: \mathbb{R}^{n} \rightarrow \mathbb{R}$ for $i=1, \ldots, k$ be fixed $C^{2}$ functions. Let $g: U \times \mathbb{R}^{n+k} \rightarrow \mathbb{R}$ be defined by

$$
g(u, x, d)=f(u, x)-\sum_{i=1}^{k} d_{i} G_{i}(x)
$$

for $u \in U, x \in \mathbb{R}^{n}$ and $d \in \mathbb{R}^{k}$. Assume that $\frac{\partial g}{\partial z_{j}}\left(u^{0}, x^{0}, d^{0}\right)=0$ for $j=$ $1, \ldots, n+k$ and

$$
\operatorname{det}\left(\frac{\partial^{2} g}{\partial z_{i} \partial z_{j}}\left(u^{0}, x^{0}, d^{0}\right)\right) \neq 0
$$

for some $\left(u^{0}, x^{0}, d^{0}\right) \in U \times \mathbb{R}^{n+k}$ and $i, j=1, \ldots, n+k$ (we do not differentiate with respect to the coordinates of $u)$. Assume that $\left(u^{m}, x^{m}, d^{m}\right) \in$ $U \times \mathbb{R}^{n+k}$ and $\left(u^{m}, y^{m}, z^{m}\right) \in U \times \mathbb{R}^{n+k}$ are such that $\left(u^{m}, x^{m}, d^{m}\right) \rightarrow$ $\left(u^{0}, x^{0}, d^{0}\right)$ and $\left(u^{m}, y^{m}, z^{m}\right) \rightarrow\left(u^{0}, x^{0}, d^{0}\right)$ with respect to any norm in 
$\mathbb{R}^{l+n+k}$. If, for any $m \in \mathbb{N}, \frac{\partial g}{\partial z_{j}}\left(u^{m}, x^{m}, d^{m}\right)=0$ and $\frac{\partial g}{\partial z_{j}}\left(u^{m}, y^{m}, z^{m}\right)=0$ for $j=1, \ldots, n+k$ then

$$
\left(u^{m}, x^{m}, d^{m}\right)=\left(u^{m}, y^{m}, z^{m}\right) \quad \text { for } m \geq m_{0} .
$$

Lemma 2.9 ([3]). Let $A \in \mathbb{R}^{n \times n}$ be a symmetric matrix. Let $\lambda_{k_{1}}, \lambda_{k_{2}}$ be eigenvalues of $A$, with $\lambda_{k_{i}}$ of multiplicity $j_{i}$ for $i=1,2$. Assume that for $i=1,2,\left\{v^{i j}: j=1, \ldots, j_{i}\right\}$ is an orthonormal basis of the eigenspace of $\lambda^{k_{i}}$. Define a $\left(j_{1}+j_{2}\right) \times n$ matrix $V$ with rows $v^{i j}, i=1,2, j=1, \ldots, j_{i}$. Let

$$
C=\left(\begin{array}{rr}
A-\lambda_{k_{1}} \text { id } & V^{T} \\
V & 0
\end{array}\right) .
$$

Then $\operatorname{det}(C) \neq 0$.

Lemma 2.10 ([3]). Assume that $t \in \mathbb{R}$, let $B, E$ be fixed $n \times n$ matrices and let $A$ be a fixed $m \times m$ matrix. Define

$$
C(t)=\left(\begin{array}{rr}
A & D \\
D_{1} & B+t E
\end{array}\right),
$$

where $D$ is a fixed $m \times n$ matrix and $D_{1}$ is a fixed $n \times m$ matrix. If $\operatorname{det}(C(t))=$ $\sum_{j=0}^{n} a_{j} t^{j}$, then

$$
a_{n}=\operatorname{det}(A) \operatorname{det}(E) .
$$

Now we prove some other technical results.

Theorem 2.11. Let $n=2$ and $N=4$. Let $z=\left(z_{2}, z_{3}, z_{4}\right)$ be such that $z_{i}= \pm 1$ for $i=2,3,4$ and $z_{j}=-1$ for exactly one $j \in\{2,3,4\}$. Let

$$
A_{z}=\left(a_{i j}(z)\right)=\left(\begin{array}{rrrr}
1 & z_{2} & z_{3} & z_{4} \\
z_{2} & 1 & -1 & -1 \\
z_{3} & -1 & 1 & -1 \\
z_{4} & -1 & -1 & 1
\end{array}\right)
$$

and

$$
\begin{array}{r}
M_{A}=\max \left\{\sum_{i, j=1}^{4} u_{i} u_{j} a_{i j}(z)\left\langle x_{i}, x_{j}\right\rangle_{2}:\left(x^{1}, x^{2}\right) \in\left(\mathbb{R}^{4}\right)^{2} \text { satisfy }[2.2),\right. \\
\left.\qquad \sum_{i=1}^{4} u_{i}^{2}=1\right\} .
\end{array}
$$

Then $M_{A}=4 / 3$.

Proof. By Lemma 2.7, we can assume that $z_{4}=-1$. Fix $u \in \mathbb{R}^{4}$ with $\sum_{i=1}^{4} u_{i}^{2}=1$. Let $B_{u}=\left(u_{i} u_{j} a_{i j}(z)\right)_{i, j=1}^{4}$. By Lemma 2.2 .

$$
M_{A}=\max \left\{b_{1}(u, A)+b_{2}(u, A): u \in \mathbb{R}^{4}, \sum_{i=1}^{4} u_{i}^{2}=1\right\} \text {, }
$$


where $b_{1}(u, A) \geq b_{2}(u, A)$ denote the two largest eigenvalues of $B_{u}$. Put $v_{i}=u_{i}^{2}$ for $i=1, \ldots, 4$. After elementary calculations (see also the file theorem 2.11.nb at our web site) we get

$$
\operatorname{det}\left(B_{u}-t \mathrm{id}\right)=t^{4}-t^{3} \sum_{i=1}^{4} v_{i}+4 t v_{3} v_{2}\left(v_{1}+v_{4}\right) .
$$

Define $w=\left(w_{1}, \ldots, w_{4}\right)$ by $w_{1}=0, w_{4}=\sqrt{u_{1}^{2}+u_{4}^{2}}, w_{i}=u_{i}$ for $i=2,3$. Observe that by the above formula, $B_{u}$ and $B_{w}$ have the same eigenvalues. Since $w_{1}=0$, by Lemmas 2.5 and 2.6 and Theorems 2.3 and 2.4 applied to $n=2$ and $N=4$ we get

$$
b_{1}(u, A)+b_{2}(u, A) \leq \lambda_{2}^{3}=4 / 3,
$$

which completes the proof.

Lemma 2.12. Let $n=2, N=4$ and $u \in[0,1 / \sqrt{3})$. Let

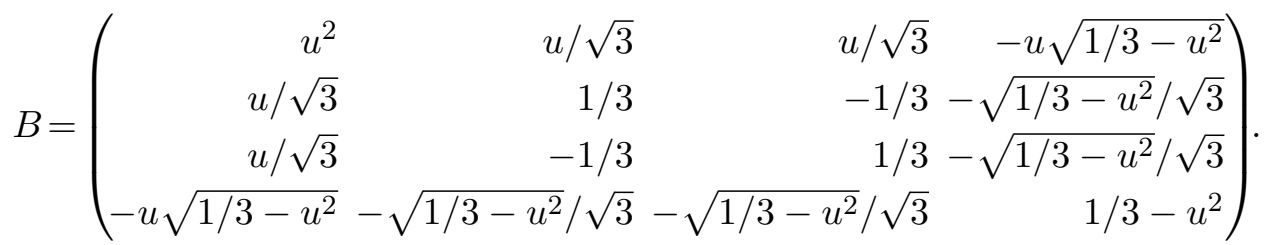

Then the eigenvalues of $B$ are $2 / 3$ (with multiplicity 2 ), $-1 / 3$ and 0 . Moreover,

$$
w^{1}=\left(\sqrt{2} u, 1 / \sqrt{6}, 1 / \sqrt{6},-\sqrt{2\left(1-3 u^{2}\right)} / \sqrt{3}\right), \quad w^{2}=(0,-1 / \sqrt{2}, 1 / \sqrt{2}, 0)
$$

are orthonormal eigenvectors corresponding to $2 / 3$, and

$$
w^{3}=\left(1,0,0, u / \sqrt{1 / 3-u^{2}}\right)
$$

is an eigenvector corresponding to 0.

Proof. Elementary calculations (see also the file lemma2.12 nb at our web site).

Lemma 2.13. Let $n=2, N=4$ and $u \in[0,1)$. Let

$$
B=\left(\begin{array}{rrrr}
u^{2} & u \sqrt{1-u^{2}} / \sqrt{2} & u \sqrt{1-u^{2}} / \sqrt{2} & 0 \\
u \sqrt{1-u^{2}} / \sqrt{2} & \left(1-u^{2}\right) / 2 & \left(u^{2}-1\right) / 2 & 0 \\
u \sqrt{1-u^{2}} / \sqrt{2} & \left(u^{2}-1\right) / 2 & \left(1-u^{2}\right) / 2 & 0 \\
0 & 0 & 0 & 0
\end{array}\right) .
$$

Then the eigenvalues of $B$ are

$$
0,\left(u^{2}+\sqrt{4 u^{2}-3 u^{4}}\right) / 2,1-u^{2},\left(u^{2}-\sqrt{4 u^{2}-3 u^{4}}\right) / 2 .
$$


Moreover,

$$
w^{2}=\left(z / \sqrt{z^{2}+2}, 1 / \sqrt{z^{2}+2}, 1 / \sqrt{z^{2}+2}, 0\right)
$$

where

$$
z=\left(u^{2}+\sqrt{4 u^{2}-3 u^{4}}\right) / u\left(\sqrt{2-2 u^{2}}\right),
$$

is an eigenvector corresponding to $\left(u^{2}+\sqrt{4 u^{2}-3 u^{4}}\right) / 2$, and

$$
w^{3}=(0,-1 / \sqrt{2}, 1 / \sqrt{2}, 0)
$$

is an eigenvector corresponding to $1-u^{2}$. Also

$$
M=\max \left\{1-u^{2}+\left(u^{2}+\sqrt{4 u^{2}-3 u^{4}}\right) / 2: u \in[1 / \sqrt{3}, 1]\right\}=4 / 3 .
$$

Proof. It can be verified by elementary calculations that the above defined numbers are the eigenvalues of $B$ (see the file lemma 2.13 nb at our web site). Also notice that if

$$
f(v)=1-v / 2+\sqrt{4 v-3 v^{2}} / 2,
$$

then

$$
f^{\prime}(v)=-1 / 2+(4-6 v) /\left(4 \sqrt{4 v-3 v^{2}}\right) .
$$

Note that $f^{\prime}(v)=0$ if and only if $3 v^{2}-4 v+1=0$. Hence $f^{\prime}(1)=f^{\prime}(1 / 3)=0$. Since $f(1)=1, M=f(1 / 3)=4 / 3$. Observe that if $u=1 / \sqrt{3}$ then $v=1 / 3$, which shows our claim.

Lemma 2.14. Let $n=2, N=4$ and $c \in[0,1 / \sqrt{3})$. Let

$$
B=\left(\begin{array}{rrrr}
1-3 c^{2} & c \sqrt{1-3 c^{2}} & c \sqrt{1-3 c^{2}} & c \sqrt{1-3 c^{2}} \\
c \sqrt{1-3 c^{2}} & c^{2} & -c^{2} & -c^{2} \\
c \sqrt{1-3 c^{2}} & -c^{2} & c^{2} & -c^{2} \\
c \sqrt{1-3 c^{2}} & -c^{2} & -c^{2} & c^{2}
\end{array}\right) .
$$

Then the eigenvalues of $B$ are $2 c^{2}$ (with multiplicity 2 ),

$$
\left(1-4 c^{2}+\sqrt{1+8 c^{2}-32 c^{4}}\right) / 2 \text { and }\left(1-4 c^{2}-\sqrt{1+8 c^{2}-32 c^{4}}\right) / 2 .
$$

Moreover,

$$
w^{1}=(0,1 / \sqrt{6}, 1 / \sqrt{6},-2 / \sqrt{6}), \quad w^{2}=(0,-1 / \sqrt{2}, 1 / \sqrt{2}, 0)
$$

are orthonormal eigenvectors corresponding to $2 c^{2}$.

Proof. Elementary calculations (see also the file lemma2.14 at our web site).

Lemma 2.15. Let $A=\left(a_{i j}\right)_{i, j=1}^{N}$ be a symmetric matrix such that $a_{i j} \in$ $\{ \pm 1\}$ for $i, j=1, \ldots, N$ and $a_{i i}=1$ for $i=1, \ldots, N$. Consider the function

$$
f_{u_{1}, A}^{N}\left(\left(u_{2}, \ldots, u_{N}\right), x^{1}, x^{2}\right)=\sum_{i, j=1}^{N} u_{i} u_{j} a_{i j}\left\langle x_{i}, x_{j}\right\rangle_{2}
$$


under constraints 2.2 and $(2.3)$. Then there exist $x^{1}, x^{2} \in \mathbb{R}^{N}$ satisfying (2.2) and $\left(u_{2}, \ldots, u_{N}\right)$ satisfying (2.3), maximizing $f_{u_{1}, A}^{N}$, and such that $x_{N-1}^{2} \geq 0, x_{N}^{2}=0$, and $x_{N-2}^{1} \geq 0$.

Proof. Let $y^{1}, y^{2}$ and $\left(u_{2}, \ldots, u_{N}\right)$ be any vectors satisfying (2.2) and 2.3 and maximizing $f_{u_{1}, A}^{N}$. Let $V=\operatorname{span}\left[y^{1}, y^{2}\right]$. Since $\operatorname{dim}(V)=2$, there exist linearly independent $f^{1}, \ldots, f^{N-2} \in \mathbb{R}^{N}$ such that $V=\bigcap_{j=1}^{N-2} \operatorname{ker}\left(f^{j}\right)$. Hence we can find $d^{2} \in V \backslash\{0\}$ orthogonal to $e_{N}$ such that $d_{N-1}^{2} \geq 0$. Set $x^{2}=d /\left\|d^{2}\right\|_{2}$. Analogously, we can find $d^{1} \in V \backslash\{0\}$ orthogonal to $x^{2}$ with $d_{N-2}^{1} \geq 0$. Set $x^{1}=d^{1} /\left\|d^{1}\right\|_{2}$. Note that $x^{i} \in V$ for $i=1,2$ and they are orthonormal. By Lemma 2.1, $x^{1}, x^{2}$ and $\left(u_{2}, \ldots, u_{N}\right)$ maximize $f_{u_{1}, A}^{N}$, which completes the proof.

In the following, for fixed $N \geq 4$, we will work with a function $f_{u_{N-3}}^{N}$ instead of $f_{u_{1}}\left(u_{N-3} \in[0,1]\right.$ is fixed). More precisely,

$$
f_{u_{N-3}}^{N}\left(\left(v_{1}, \ldots, v_{N-4}, v_{N-2}, v_{N-1}, v_{N}\right), z^{1}, z^{2}\right)=\sum_{i, j=1}^{N} v_{i} v_{j}\left|\left\langle z_{i}, z_{j}\right\rangle_{2}\right|,
$$

where $v_{N-3}=u_{N-3}$. Also define, for any $N \times N$ matrix $A$,

$$
\begin{aligned}
f_{u_{N-3}, A}^{N}\left(\left(v_{1}, \ldots, v_{N-4}, v_{N-2}, v_{N-1}, v_{N}\right), z^{1}, z^{2}\right) & \\
& =\sum_{i, j=1}^{N} a_{i j} v_{i} v_{j}\left\langle z_{i}, z_{j}\right\rangle_{2} .
\end{aligned}
$$

The next three theorems show what candidates for maximizing $f_{u_{N-3}}^{N}$ look like.

Theorem 2.16. Let $A$ be an $N \times N$ symmetric matrix defined by

$$
A=\left(\begin{array}{rrrrrrr}
1 & a_{1,2} & \ldots & a_{1, N-3} & a_{1, N-2} & a_{1, N-1} & a_{1, N} \\
\ldots & \ldots & \ldots & \ldots & \ldots & \ldots & \ldots \\
a_{N-3,1} & a_{N-3,2} & \ldots & 1 & 1 & 1 & a_{N-3, N} \\
a_{N-2,1} & a_{N-2,2} & \ldots & 1 & 1 & -1 & a_{N-2, N} \\
a_{N-1,1} & a_{N-1,2} & \ldots & 1 & -1 & 1 & a_{N-1, N} \\
a_{N, 1} & a_{N, 2} & \ldots & a_{N, N-3} & a_{N, N-2} & a_{N, N-1} & 1
\end{array}\right),
$$

where $a_{i j} \in\{-1,1\}$ for $i \neq j$. Assume additionally that

$$
a_{j, N}=a_{N, j}=-1
$$

for $j=N-3, N-2, N-1$. Fix $t \in \mathbb{R}$ and $u_{N-3} \in[0,1 / \sqrt{3})$. Define 
$h_{u_{N-3}, A, t}^{N}: \mathbb{R}^{N-1} \times\left(\mathbb{R}^{N}\right)^{2} \times \mathbb{R}^{3} \times \mathbb{R} \rightarrow \mathbb{R}$ by

$$
\begin{aligned}
h_{u_{N-3}, A, t}^{N} & \left(\left(v_{1}, \ldots, v_{N-4}, v_{N-2}, v_{N-1}, v_{N}\right), z^{1}, z^{2}, b_{1}, b_{2}, b_{12}, b_{4}\right) \\
= & f_{u_{N-3}, A}^{N}\left(\left(v_{1}, \ldots, v_{N-4}, v_{N-2}, v_{N-1}, v_{N}\right), z^{1}, z^{2}\right) \\
& +t\left(\left(v_{N-1}+v_{N-2}\right) / \sqrt{1-3 u_{N-3}^{2}}+v_{N}+z_{N-1}^{2}-z_{N-2}^{2}\right) \\
& -\left(b_{1}\left(\left\langle z^{1}, z^{1}\right\rangle_{N}-1\right)+b_{2}\left(\left\langle z^{2}, z^{2}\right\rangle_{N}-1\right)\right) \\
& -b_{12}\left\langle z^{1}, z^{2}\right\rangle_{N}-b_{4}\left(\left\langle\left(u_{N-3}, v\right),\left(u_{N-3}, v\right)\right\rangle_{N}-1\right),
\end{aligned}
$$

where $v=\left(v_{1}, \ldots, v_{N-4}, v_{N-2}, v_{N-1}, v_{N}\right)$. For fixed $N \in \mathbb{N}$, define

$$
\begin{aligned}
u^{N}=u & =\left(0, \ldots, 0_{N-4}, u_{N-2}, u_{N-1}, u_{N}\right), \\
x^{1 N}=x^{1} & =\left(0, \ldots, 0_{N-4}, x_{N-3}^{1}, x_{N-2}^{1}, x_{N-1}^{1}, x_{N}^{1}\right), \\
x^{2 N} & =x^{2}=\left(0, \ldots, 0_{N-4}, x_{N-3}^{2}, x_{N-2}^{2}, x_{N-1}^{2}, x_{N}^{2}\right)
\end{aligned}
$$

and

$$
d^{N}=d=\left(d_{1}, d_{2}, d_{12}, d_{4}\right) .
$$

Here $u_{N-2}=u_{N-1}=1 / \sqrt{3}, u_{N}=\sqrt{1 / 3-u_{N-3}^{2}}, x_{N-3}^{1}=\sqrt{2} u_{N-3}, x_{N-2}^{1}=$ $x_{N-1}^{1}=1 / \sqrt{6}, x_{N}^{1}=-\sqrt{2\left(1-3 u_{N-3}^{2}\right)} / \sqrt{3}, x_{N-3}^{2}=0, x_{N-2}^{2}=-x_{N-1}^{2}=$ $-1 / \sqrt{2}, x_{N}^{2}=0, d_{1}=2 / 3, d_{2}=2 / 3+t / \sqrt{2}, d_{12}=0$ and $d_{4}=4 / 3+$ $t /\left(2 \sqrt{1 / 3-u_{N-3}^{2}}\right)$. Then

$$
\frac{\partial h_{u_{N-3}, A, t}^{N}}{\partial w_{j}}\left(x^{1}, x^{2}, u, d\right)=0
$$

for $j=1, \ldots, 3 N+3$ where

$$
\begin{array}{r}
w_{j} \in\left\{v_{1}, \ldots, v_{N-4}, v_{N-2}, v_{N-1}, v_{N}, z_{k}^{i}(i=1,2, k=1, \ldots, N)\right\} \\
j=1, \ldots, 3 N-1,
\end{array}
$$

and

$$
w_{j} \in\left\{b_{12}, b_{1}, b_{2}, b_{4}\right\}, \quad j=3 N, \ldots, 3 N+3 .
$$

( We do not differentiate with respect to $u_{N-3}$. )

Proof. Notice that for

$$
w_{j} \in\left\{z_{k}^{i}: i=1,2, k=1, \ldots, N\right\}
$$

the equation (E) follows from the fact that (for $N=4$ ) $x^{i 4}=x^{i}, i=1,2$, are orthonormal eigenvectors of the matrix $B$ defined by $(2.12)$ corresponding to the eigenvalues $d_{i}, i=1,2$, which has been established in Lemma 2.12 . Also for

$$
w_{j} \in\left\{b_{12}, b_{1}, b_{2}, b_{4}\right\}
$$

the equation (E) follows immediately from the fact that $\left\langle x^{i}, x^{j}\right\rangle_{N}=\delta_{i j}$ for $i, j=1,2, i \leq j$ and $\left\langle\left(u_{N-3}, u\right),\left(u_{N-3}, u\right)\right\rangle_{N}=1$. 
To end the proof, we show that (E) holds for

$$
w_{j} \in\left\{v_{1}, \ldots, v_{N-4}, v_{N-2}, v_{N-1}, v_{N}\right\} .
$$

Notice that, for $i=1, \ldots, N-4$,

$$
\frac{\partial h_{u_{N-3}, A, t}^{N}}{\partial v_{i}}\left(x^{1}, x^{2}, u, d\right)=2 \sum_{j=1}^{N} u_{j} a_{i j}\left\langle x_{i}, x_{j}\right\rangle_{2}-2 u_{i} d_{4}=0
$$

since $x_{i}=0$ and $u_{i}=0$ for $i=1, \ldots, N-4$. Now assume that $w_{j}=v_{N-2}$. Then the derivative equals

$$
\begin{aligned}
& 2 \sum_{j=1}^{N} u_{j} a_{N-2, j}\left\langle x_{N-2}, x_{j}\right\rangle_{2}+t / \sqrt{1-3 u_{N-3}^{2}}-2 u_{N-2} d_{4} \\
& =2\left(u_{N-3}^{2} / \sqrt{3}+1 / \sqrt{3}+(1 / 3 \sqrt{3})\left(1-3 u_{N-3}^{2}\right)+t / 2 \sqrt{1-3 u_{N-3}^{2}}-u_{N-2} d_{4}\right) \\
& =2\left((4 / 3) / \sqrt{3}+t / 2 \sqrt{1-3 u_{N-3}^{2}}-(4 / 3) u_{N-2}-\left(t / 2 \sqrt{1 / 3-u_{N-3}^{2}}\right) u_{N-2}\right) \\
& =0 .
\end{aligned}
$$

The same calculation works for $i=N-1$. If $i=N$, then we obtain

$$
\begin{aligned}
2 \sum_{j=1}^{N} u_{j} a_{N, j}\left\langle x_{N},\right. & \left.x_{j}\right\rangle_{2}+t-2 u_{N} d_{4} \\
= & 2\left(2 u_{N-3}^{2} \sqrt{1-3 u_{N-3}^{2}} / \sqrt{3}+2 \sqrt{1-3 u_{N-3}^{2}} / 3 \sqrt{3}+t / 2\right. \\
& \left.+(2 / 3)\left(1-3 u_{N-3}^{2}\right) \sqrt{1 / 3-u_{N-3}^{2}}-u_{N} d_{4}\right) \\
= & 2\left(2 u_{N-3}^{2} \sqrt{1 / 3-u_{N-3}^{2}}+(4 / 3) \sqrt{1 / 3-u_{N-3}^{2}}+t / 2\right. \\
& \left.-2 u_{N-3}^{2} \sqrt{1 / 3-u_{N-3}^{2}}-d_{4} u_{N}\right)=0
\end{aligned}
$$

which completes the proof.

Theorem 2.17. Let $A$ be as in 2.18). Fix $t \in \mathbb{R}$ and $u_{N-3} \in[1 / \sqrt{3}, 1)$. Define $h_{u_{N-3}, A, t}^{N}: \mathbb{R}^{N-1} \times\left(\mathbb{R}^{N}\right)^{2} \times \mathbb{R}^{3} \times \mathbb{R} \rightarrow \mathbb{R}$ by

$$
\begin{aligned}
h_{u_{N-3}, A, t}^{N}\left(\left(v_{1}, \ldots, v_{N-4}, v_{N-2}, v_{N-1}, v_{N}\right), z^{1}, z^{2}, b_{1}, b_{2}, b_{12}, b_{4}\right) \\
=\sum_{i, j=1}^{N} a_{i j} v_{i} v_{j}\left\langle z_{i}, z_{j}\right\rangle_{2}+t\left(u_{N-2}+u_{N-1}+z_{N-1}^{2}-z_{N-2}^{2}\right) \\
\left.\quad-\left(b_{1}\left\langle z^{1}, z^{1}\right\rangle_{N}-1\right)+b_{2}\left(\left\langle z^{2}, z^{2}\right\rangle_{N}-1\right)\right) \\
\quad-b_{12}\left\langle z^{1}, z^{2}\right\rangle_{N}-b_{4}\left(\left\langle\left(u_{N-3}, v\right),\left(u_{N-3}, v\right)\right\rangle_{N}-1\right),
\end{aligned}
$$


where $v=\left(v_{1}, \ldots, v_{N-4}, v_{N-2}, v_{N-1}, v_{N}\right)$. For fixed $N \in \mathbb{N}$, define

$$
\begin{aligned}
u^{N}=u & =\left(0, \ldots, 0_{N-4}, u_{N-2}, u_{N-1}, u_{N}\right), \\
x^{1 N}=x^{1} & =\left(0, \ldots, 0_{N-4}, x_{N-3}^{1}, x_{N-2}^{1}, x_{N-1}^{1}, x_{N}^{1}\right), \\
x^{2 N}=x^{2} & =\left(0, \ldots, 0_{N-4}, x_{N-3}^{2}, x_{N-2}^{2}, x_{N-1}^{2}, x_{N}^{2}\right)
\end{aligned}
$$

and

$$
d^{N}=d=\left(d_{1}, d_{2}, d_{12}, d_{4}\right) .
$$

Here $u_{N-2}=u_{N-1}=\sqrt{\left(1-u_{N-3}^{2}\right) / 2}, u_{N}=0, x_{N-3}^{1}=0, x_{N-2}^{1}=-x_{N-1}^{1}$ $=-1 / \sqrt{2}, x_{N}^{1}=0, x_{N-2}^{2}=x_{N-1}^{2}=1 / \sqrt{2+w^{2}}, x_{N-3}^{2}=w / \sqrt{2+w^{2}}$, $x_{N}^{2}=0, d_{1}=1-u_{N-3}^{2}$,

$$
d_{2}=\left(u_{N-3}^{2}+\sqrt{4 u_{N-3}^{2}-3 u_{N-3}^{4}}\right) / 2+t / \sqrt{2},
$$

$d_{12}=0$ and $d_{4}=1+\frac{u_{N-3} w}{u_{N-2}\left(2+w^{2}\right)}+t /\left(2 u_{N-1}\right)$, where

$$
w=\frac{u_{N-3}^{2}+\sqrt{4 u_{N-3}^{2}-3 u_{N-3}^{3}}}{u_{N-3} \sqrt{2-2 u_{N-3}^{2}}} .
$$

Then

$$
\frac{\partial h_{u_{N-3}, A, t}^{N}}{\partial w_{j}}\left(x^{1}, x^{2}, u, d\right)=0
$$

for $j=1, \ldots, 3 N+3$ where

$$
\begin{array}{r}
w_{j} \in\left\{v_{1}, \ldots, v_{N-4}, v_{N-2}, v_{N-1}, v_{N}, z_{k}^{i}(i=1,2, k=1, \ldots, N)\right\}, \\
j=1, \ldots, 3 N-1,
\end{array}
$$

and

$$
w_{j} \in\left\{b_{12}, b_{1}, b_{2}, b_{4}\right\}, \quad j=3 N, \ldots, 3 N+3 .
$$

( We do not differentiate with respect to $u_{N-3}$.)

Proof. Notice that for

$$
w_{j} \in\left\{z_{k}^{i}: i=1,2, k=1, \ldots, N\right\}
$$

the equation (E) follows from the fact that (for $N=4$ ) $x^{i 4}=x^{i}, i=1,2$, are orthonormal eigenvectors of the matrix $B$ defined by $(2.13)$ corresponding to the eigenvalues $d_{i}, i=1,2$, which has been established in the proof of Lemma 2.13. Also for

$$
w_{j} \in\left\{b_{12}, b_{1}, b_{2}, b_{4}\right\}
$$

the equation (E) follows immediately from the fact that $\left\langle x^{i}, x^{j}\right\rangle_{N}=\delta_{i j}$ for $i, j=1,2, i \leq j$, and $\left\langle\left(u_{N-3}, u\right),\left(u_{N-3}, u\right)\right\rangle_{N}=1$.

To end the proof, we show that (E) holds for

$$
w_{j} \in\left\{v_{1}, \ldots, v_{N-4}, v_{N-2}, v_{N-1}, v_{N}\right\} .
$$


Notice that for $i=1, \ldots, N-4, N$,

$$
\frac{\partial h_{u_{N-3}, t}}{\partial v_{i}}\left(x^{1}, x^{2}, u, d\right)=2 \sum_{j=1}^{N} u_{j} a_{i j}\left\langle x_{i}, x_{j}\right\rangle_{2}+t-2 u_{i} d_{4}=0
$$

since $x_{i}=0$ and $u_{i}=0$ for $i=1, \ldots, N-4, N$. Now assume that $w_{j}=v_{N-2}$. Then the derivative equals

$$
\begin{aligned}
2\left(\sum_{j=1}^{N} u_{j} a_{N-2, j}\right. & \left.\left\langle x_{N-2}, x_{j}\right\rangle_{2}+t / 2-u_{N-2} d_{4}\right) \\
= & 2\left(\left(u_{N-3} w\right) /\left(2+w^{2}\right)+u_{N-2}+t / 2-u_{N-2} d_{4}\right)=0 .
\end{aligned}
$$

Since $u_{N-2}=u_{N-1}$, the same calculations work for $i=N-1$, which completes the proof.

Reasoning as in Theorems 2.16 and 2.17 and applying Lemma 2.14 we can show

Theorem 2.18. Let $A$ be as in 2.18). Assume additionally that

$$
1=a_{N, N-3}=-a_{N, N-2}=-a_{N, N-1} .
$$

Fix $t \in \mathbb{R}$ and $u_{N-3} \in[0,1)$. Define $h_{u_{N-3}, A, t}^{N}: \mathbb{R}^{N-1} \times\left(\mathbb{R}^{N}\right)^{2} \times \mathbb{R}^{3} \times \mathbb{R} \rightarrow \mathbb{R}$ by

$$
\begin{aligned}
h_{u_{N-3}, A, t}^{N} & \left(\left(v_{1}, \ldots, v_{N-4}, v_{N-2}, v_{N-1}, v_{N}\right), z^{1}, z^{2}, b_{1}, b_{2}, b_{12}, b_{4}\right) \\
= & \sum_{i, j=1}^{N} a_{i j} v_{i} v_{j}\left\langle z_{i}, z_{j}\right\rangle_{2}+t\left(u_{N}+u_{N-2}+u_{N-1}+z_{N-1}^{2}-z_{N-2}^{2}\right) \\
& \quad-\left(b_{1}\left(\left\langle z^{1}, z^{1}\right\rangle_{N}-1\right)+b_{2}\left(\left\langle z^{2}, z^{2}\right\rangle_{N}-1\right)\right) \\
& -b_{12}\left\langle z^{1}, z^{2}\right\rangle_{N}-b_{4}\left(\left\langle\left(u_{N-3}, v\right),\left(u_{N-3}, v\right)\right\rangle_{N}-1\right),
\end{aligned}
$$

where $v=\left(v_{1}, \ldots, v_{N-4}, v_{N-2}, v_{N-1}, v_{N}\right)$. For fixed $N \in \mathbb{N}$, define

$$
\begin{aligned}
u^{N}=u & =\left(0, \ldots, 0_{N-4}, u_{N-2}, u_{N-1}, u_{N}\right), \\
x^{1 N}=x^{1} & =\left(0, \ldots, 0_{N-4}, x_{N-3}^{1}, x_{N-2}^{1}, x_{N-1}^{1}, x_{N}^{1}\right), \\
x^{2 N}=x^{2} & =\left(0, \ldots, 0_{N-4}, x_{N-3}^{2}, x_{N-2}^{2}, x_{N-1}^{2}, x_{N}^{2}\right)
\end{aligned}
$$

and

$$
d^{N}=d=\left(d_{1}, d_{2}, d_{12}, d_{4}\right) .
$$

Here $u_{N-2}=u_{N-1}=u_{N}=\sqrt{\left(1-u_{N-3}^{2}\right) / 3}, x_{N-2}^{1}=x_{N-1}^{1}=1 / \sqrt{6}, x_{N}^{1}=$ $-2 / \sqrt{6}, x_{N-2}^{2}=-x_{N-1}^{2}=-1 / \sqrt{2}, x_{N}^{2}=0 d_{1}=2 c^{2}, d_{2}=2 c^{2}+t / \sqrt{2}$ $d_{12}=0$ and $d_{4}=4 c^{2}+t /\left(2 u_{N}\right)$, where $c=\sqrt{\left(1-u_{N-3}^{2}\right) / 3}$. Then

$$
\frac{\partial h_{u_{N-3}, A, t}^{N}}{\partial w_{j}}\left(x^{1}, x^{2}, u, d\right)=0
$$


for $j=1, \ldots, 3 N+3$ where

$$
\begin{aligned}
w_{j} \in\left\{v_{1}, \ldots, v_{N-4}, v_{N-2}, v_{N-1}, v_{N}, z_{k}^{i}(i=1,2, k\right. & =1, \ldots, N)\}, \\
j & =1, \ldots, 3 N-1,
\end{aligned}
$$

and

$$
w_{j} \in\left\{b_{12}, b_{1}, b_{2}, b_{4}\right\}, \quad j=3 N, \ldots, 3 N+3 .
$$

(We do not differentiate with respect to $u_{N-3}$.)

Lemma 2.19. Let $A$ be as in Theorem 2.16. For fixed $u_{N-3} \in[0,1 / \sqrt{3})$ and $t>0$ define $g_{u_{N-3}, A, t}^{N}: \mathbb{R}^{N-1} \times\left(\mathbb{R}^{N}\right)^{2} \rightarrow \mathbb{R}$ by

$$
\begin{aligned}
g_{u_{N-3}, A, t}^{N}\left(\left(v_{1}, \ldots, v_{N-4}, v_{N-2}, v_{N-1}, v_{N}\right), y^{1}, y^{2}\right) & =\sum_{i, j=1}^{N} v_{i} v_{j} a_{i j}\left\langle y_{i}, y_{j}\right\rangle_{2} \\
& +g_{u_{N-3}}^{1, N}\left(\left(v_{1}, \ldots, v_{N-4}, v_{N-2}, v_{N-1}, v_{N}\right), y^{1}, y^{2}\right),
\end{aligned}
$$

where $v_{N-3}=u_{N-3}$ is fixed, and

$$
\begin{aligned}
g_{u_{N-3}}^{1, N}\left(\left(v_{1}, \ldots,\right.\right. & \left.\left.v_{N-4}, v_{N-2}, v_{N-1}, v_{N}\right), y^{1}, y^{2}\right) \\
& =\left(v_{N}+\left(v_{N-2}+v_{N-1}\right) / \sqrt{1-3 v_{N-3}^{2}}+y_{N-1}^{2}-y_{N-2}^{2}\right) .
\end{aligned}
$$

Let $M_{u_{N-3}, A, t}^{N}=\max g_{u_{N-3}, A, t}^{N}$ under the constraints

$$
\left\langle y^{i}, y^{j}\right\rangle_{N}=\delta_{i j}, \quad 1 \leq i \leq j \leq 2, \quad \sum_{j=1}^{N} v_{j}^{2}=1 .
$$

Assume that $u_{N-3} \in[0,1 / \sqrt{3})$ is so chosen that

$$
M_{u_{N-3}, A, 0}^{N}=g_{u_{N-3}, A, 0}\left(u^{N}, x^{1}, x^{2}\right),
$$

where $u^{N}, x^{1}, x^{2}$ are as in Theorem 2.16. Set

$$
D_{u_{N-3}}^{N}=\left\{\left(v_{1}, \ldots, v_{N-4}, v_{N-2}, v_{N-1}, v_{N}, y^{1}, y^{2}\right): y_{N}^{2}=0, y_{N-2}^{1} \geq 0\right\} .
$$

Then

$$
X_{u_{N-3}}^{N}=\left(u^{N}, x^{1}, x^{2}\right)
$$

is the only point maximizing $g_{u_{N-3}, A, t}^{N}$ satisfying 2.2 and 2.3 belonging to $D_{u_{N-3}}^{N}$.

Proof. Let

$$
Y_{u_{N-3}}^{N}=\left(\left(v_{1}, \ldots, v_{N-4}, v_{N-2}, v_{N-1}, v_{N}\right), y^{1}, y^{2}\right) \in D_{u_{N-3}}^{N}
$$

maximize $g_{u_{N-3}, A, t}^{N}$ and satisfy 2.2 and 2.3. Notice that $g_{u_{N-3}}^{1, N}$ (as a function of $v=\left(v_{1}, \ldots, v_{N-4}, v_{N-2}, \ldots, v_{n}\right)$ and $\left.y^{2}\right)$ attains its maximum under 
constraints 2.2 and 2.3 only at

$$
v=\left(0, \ldots, 0_{N-4}, 1 / \sqrt{3}, 1 / \sqrt{3}, \sqrt{1 / 3-u_{N-3}^{2}}\right)
$$

and

$$
y^{2}=\left(0, \ldots, 0_{N-3},-1 / \sqrt{2}, 1 / \sqrt{2}, 0\right) .
$$

Since $g_{u_{N-3}}^{1, N}$ does not depend on $y^{1}, t>0$, and the maximum of $g_{u_{N-3}, A, 0}^{N}$ is attained at $X_{u_{N-3}}^{N}$, we have $v_{i}=0$ for $i=1, \ldots, N-4, v_{N-2}=v_{N-1}=$ $1 / \sqrt{3}, v_{N}=u_{N}$ and $y^{2}=x^{2}$. Since $x^{1}, x^{2}$ are eigenvectors of $A$, by Lemma 2.2. $\operatorname{span}\left[y^{i}: i=1,2\right]=\operatorname{span}\left[x^{i}: i=1,2\right]$. Note that

$$
\left\langle x^{1}, x^{2}\right\rangle_{N}=\left\langle y^{1}, x^{2}\right\rangle_{N}=0 .
$$

Hence $y^{1}=d x^{1}$. Since $\left\langle y^{1}, y^{1}\right\rangle_{N}=1, y_{N-2}^{1} \geq 0$ and $x_{N-2}^{1}>0$, it follows that $x^{1}=y^{1}$, as required.

REMARK 2.20. Lemma 2.19 remains true (with the same proof) if we replace the function $g_{u_{N-3}}^{1, N}$ by

$g_{u_{N-3}}^{2, N}\left(\left(v_{1}, \ldots, v_{N-4}, v_{N-2}, v_{N-1}, v_{N}\right), y^{1}, y^{2}\right)=v_{N-2}+v_{N-1}+y_{N-2}^{2}-y_{N-1}^{2}$, and $X_{u_{N-3}}^{N}$ and $A$ from Theorem 2.16 by $X_{u_{N-3}}^{N}$ and $A$ from Theorem 2.17 Also the statement of Lemma 2.19 remains true if we replace $g_{u_{N-3}}^{1, N}$ by

$$
\begin{aligned}
g_{u_{N-3}}^{3, N}\left(\left(v_{1}, \ldots, v_{N-4}, v_{N-2}, v_{N-1}, v_{N}\right), y^{1}, y^{2}\right) & \\
& =v_{N-2}+v_{N-1}+v_{N}+y_{N-2}^{2}-y_{N-1}^{2},
\end{aligned}
$$

and $X_{u_{N-3}}^{N}$ and $A$ from Theorem 2.16 by those from Theorem 2.18

Now we demonstrate two crucial technical results for our proof of the Grünbaum conjecture.

Theorem 2.21. Fix $N \geq 4$ and $u_{N-3} \in[0,1 / \sqrt{3})$. Let $A$ and

$$
X_{u_{N-3}}^{N}=\left(u^{N}, x^{1}, x^{2}, d=d(t)\right)
$$

be as in Theorem 2.16, Let

$$
M_{u_{N-3}, A, t}^{N}=\max g_{u_{N-3}, A, t}^{N},
$$

where $g_{u_{N-3}, A, t}^{N}$ has been defined in Lemma 2.19, under the constraints

$$
\left\langle y^{i}, y^{j}\right\rangle_{N}=\delta_{i j}, \quad 1 \leq i \leq j \leq 2, \quad \sum_{j=1, j \neq N-3}^{N} v_{j}^{2}=1-u_{N-3}^{2} .
$$

Assume that $u_{N-3} \in[0,1 / \sqrt{3})$ is so chosen that

$$
M_{u_{N-3}, A, 0}^{N}=f_{u_{N-3}, A}^{N}\left(X_{u_{N-3}}^{N}\right) .
$$


Denote by $D_{u, A, t}^{N}$ the $(3 N+2) \times(3 N+2)$ matrix defined by

$$
D_{u, A, t}^{N}=\left(\frac{\partial^{2}\left(h_{u_{N-3}, A, t}^{N}\right)}{\partial w_{i} \partial w_{j}}\left(x^{1}, x^{2}, u, d_{1}(t), d_{2}(t), d_{12}(t), d_{4}(t)\right)\right)_{i, j=1}^{3 N+2},
$$

where

$$
\begin{aligned}
w_{i}, w_{j} \in\{ & \left.v_{1}, \ldots, v_{N-4}, v_{N-2}, v_{N-1}, v_{N}, y_{j}^{1}(j=1, \ldots, N)\right\} \\
& \cup\left\{y_{j}^{2}(j=1, \ldots, N-1), b_{1}, b_{2}, b_{1,2}, b_{4}\right\}
\end{aligned}
$$

(we do not differentiate with respect to $u_{N-3}$ and $y_{N}^{2}$ ). Assume that

$$
\operatorname{det}\left(D_{u, A, t}^{N}\right)=\sum_{j=o}^{k} c_{j, N}(u) t^{j}
$$

and $c_{j, N}\left(u_{N-3}\right) \neq 0$ for some $j \in\{1, \ldots, k\}$. Then there exists an open interval $U_{N} \subset[0,1 / \sqrt{3})\left(U_{N}=[0, w)\right.$ if $\left.u_{N-3}=0\right)$ such that $u_{N-3} \in U_{N}$ and for any $u \in U_{N}$ the function $f_{u, A}^{N}$ attains its global maximum under constraints (2.2) and 2.3) at $\left(u^{N}, x^{1}, x^{2}\right)$ (corresponding to $u$ ) defined in Theorem 2.16. The same result holds true if we replace the function $g_{u, A, t}^{N}$ from Theorem 2.16 by the one from Theorem 2.17 and we assume that $u_{N-3} \in[1 / \sqrt{3}, 1)$. In this case $\left(x^{1}, x^{2}, u^{N}, d_{1}(t), d_{2}(t), d_{12}(t), d_{4}(t)\right)$ are as in Theorem 2.17 .

Proof. Fix $N \geq 4$ and $u_{N-3} \in[0,1 / \sqrt{3})$ satisfying our assumptions. Let $j_{0}=\min \left\{j \in\{0, \ldots, 2(N-4)+4\}: c_{j, N}\left(u_{N-3}\right) \neq 0\right\}$. For $(u, t) \in[0,1) \times \mathbb{R}$, set

$$
h(t, u)=\sum_{j=j_{0}}^{2(N-4)+4} c_{j, N}(u) t^{j-j_{0}} .
$$

Since $c_{j_{0}, N}\left(u_{N-3}\right) \neq 0$, and $c_{j, N}$ are continuous, there exists an open interval $U \subset[0,1 / \sqrt{3})$ and $\delta>0$ such that $u_{N-3} \in U$ and

$$
h(t, u) \neq 0
$$

for $u \in U$ and $|t|<\delta$. Fix $t_{0} \in(0, \delta)$. Set

$$
U_{t_{0}}=\left\{u \in U: M_{u, A, t_{0}}^{N}=g_{u, A, t_{0}}^{N}\left(X_{u}^{N}\right)\right\} .
$$

Note that $u_{N-3} \in U_{t_{0}}$.

Now we show that $U_{t_{0}}$ is an open set. Let $u_{0} \in U_{t_{0}}$. Assume on the contrary that there exist $z_{n} \in U \backslash U_{t_{0}}$ such that $z_{n} \rightarrow u_{0}$. For any $u \in U$, let

$$
Z_{u, t_{0}}=Z_{u}=\left(v_{1, u}, \ldots, v_{N-4, u}, v_{N-2, u}, v_{N-1, u}, v_{N, u}, x^{1 u}, x^{2 u}, x^{3 u}\right)
$$

be a point maximizing $g_{u, A, t_{0}}^{N}$ under constraints 2.2 and $(2.3)$. Since the function $\left(f_{u, A}^{N}-g_{u, A, t_{0}}^{N}\right)\left(\left(v_{1}, \ldots, v_{N-4}, v_{N-2}, v_{N-1}, v_{N}\right), z^{1}, z^{2}\right)$ is independent of $z^{1}$, and by Lemma 2.15, without loss of generality, the function 
$g_{u, A, t_{0}}^{N}$ can be considered as a function of $3 N+2$ variables (we can put $\left.z_{N}^{2}=0\right)$. Consequently, we can assume that

$$
Z_{u} \in D_{u}^{N}
$$

(see (2.20)). By (2.2) and (2.3), passing to a convergent subsequence if necessary, we find that $Z_{z_{n}} \rightarrow Z$. By definition of $D_{u_{0}}^{N}, Z \in D_{u_{0}}^{N}$. Also by the continuity of the function

$$
\begin{aligned}
(v, Y) \mapsto & \sum_{i, j=1}^{N} v_{i} v_{j} a_{i j}\left\langle y_{i}, y_{j}\right\rangle_{2} \\
& +t_{0}\left(\left(v_{N-1}+v+{ }_{N-2}\right) / \sqrt{1-3 u_{n}^{2}}+v_{N}+y_{N-1}^{2}-y_{N-2}^{2}\right)
\end{aligned}
$$

we have

$$
g_{u_{0}, A, t_{0}}(Z)=M_{u_{0}, A, t_{0}} .
$$

By Lemma 2.19, $X_{u_{0}}^{N}$ is the only point in $D_{u_{0}}^{N}$ which maximizes $g_{u, A, t_{0}}^{N}$, and $Z \in D_{u_{0}}^{N}$. Hence $Z=X_{u_{0}}^{N}$. Moreover, since $X_{u_{0}}^{N} \in \operatorname{int}\left(D_{u_{0}}^{N}\right)$, by the Lagrange Multiplier Theorem, there exists

$$
M_{z_{n}}=M_{z_{n}}\left(t_{0}\right)=\left(d_{1}^{n}, d_{2}^{n}, d_{12}^{n}, d_{4}^{n}\right) \in \mathbb{R}^{4}
$$

such that

$$
\frac{\partial h_{u, A, t_{0}}^{N}}{\partial w_{i}}\left(Z_{z_{n}}, M_{z_{n}}\right)=0
$$

for $w_{i} \in X \cup D D$; here $h_{u, A, t}^{N}$ is defined by 2.19 (see Theorem 2.16 and

$$
D D=\left\{d_{1}, d_{2}, d_{12}, d_{4}\right\}
$$

Also by (2.2), 2.3), the proof of Lemma 2.2 given in [3] and 2.22,

$$
M_{z_{n}} \rightarrow L_{u_{0}}=L_{u_{0}}\left(t_{0}\right)=\left(d_{1}, d_{2}, d_{12}, d_{4}\right),
$$

where $L_{u_{0}}$ is defined in Theorem 2.16 for $t=t_{0}$ and $u_{N-3}=u_{0}$. Now we apply Lemma 2.8. Define $G: U \times \mathbb{R}^{2 N-1} \times \mathbb{R}^{N-1} \times \mathbb{R}^{4} \rightarrow \mathbb{R}^{3 N+2}$ by

$$
G(u, x, v, Q)=\left(\frac{\partial h_{u, A, t_{0}}}{\partial w_{1}}(u, x, v, Q), \ldots, \frac{\partial h_{t_{0}, u}}{\partial w_{3 N+2}}(u, x, v, Q)\right) / t_{0}^{j_{0} /(3 N+2)}
$$

for $w_{i} \in X \cup D D$. Notice that by 2.22,

$$
G\left(z_{n}, Z_{z_{n}}, M_{z_{n}}\right)=0 .
$$

Also $G\left(z_{n}, X_{z_{n}}, L_{z_{n}}\left(t_{0}\right)\right)=0$, where $\left(X_{z_{n}}, L_{z_{n}}\left(t_{0}\right)\right)$ are defined for $z_{n}$ and $t_{0}$ in Theorem 2.16. Moreover,

$$
\left(z_{n}, Z_{z_{n}}, M_{z_{n}}\right) \rightarrow\left(u_{0}, X_{u_{0}}, L_{u_{0}}\right) \quad \text { and } \quad\left(z_{n}, X_{z_{n}}, L_{z_{n}}\right) \rightarrow\left(u_{0}, X_{u_{0}}, L_{u_{0}}\right) .
$$


Notice that

$$
\begin{aligned}
\operatorname{det}\left(\frac{\partial G}{\partial w_{j}}\left(u_{0}, X_{u_{0}}, L_{u_{0}}\right)\right) & =\frac{\operatorname{det}\left(D_{u_{0}, A, t_{0}}\right)}{\left(t_{0}^{j_{0} /(3 N+2)}\right)^{3 N+2}} \\
& =\sum_{j=j_{0}}^{k} c_{j, N}\left(u_{0}\right) t_{0}^{j-j_{0}}=h\left(t_{0}, u_{0}\right) \neq 0,
\end{aligned}
$$

by definition of $j_{0}$ and $t_{0}$. By Lemma 2.8 applied to the function $G$, we have $Z_{z_{n}}=X_{z_{n}}$ and $M_{z_{n}}=L_{z_{n}}$ for $n \geq n_{0}$. Hence $z_{n} \in U_{t_{0}}$ for $n \geq n_{0}$, a contradiction. This shows that $U_{t_{0}}$ is an open set.

It is clear that $U_{t_{0}}$ is closed. Since $u_{N-3} \in U_{t_{0}}$ and $U$ is connected, $U_{t_{0}}=U$. Consequently, for any $n \in \mathbb{N}$ with $n \geq n_{0}$ and $u \in U$, the functions $g_{u, A, 1 / n}^{N}$ achieve their maximum at $u_{1}, \ldots, u_{N-4}, u_{N-2}, \ldots,, u_{N-1}, u_{N}, x^{1}, x^{2}$ (corresponding to $u_{N-3}=u_{0}$ ) defined in Theorem 2.16. Since $g_{u, A, 1 / n}^{N}$ tends uniformly to $g_{u, A, 0}^{N}=f_{u, A}^{N}$, on the set defined by 2.2 and 2.3 , with $u \in$ $U$ fixed, $f_{u, A}^{N}$ attains its maximum at $u_{1}, \ldots, u_{N-4}, u_{N-2}, u_{N-1}, u_{N}, x^{1}, x^{2}$ defined in Theorem 2.16 for any $u \in U$.

By Theorem 2.17, reasoning exactly in the same way as above we can deduce our conclusion for the function $f_{u, A}^{N}$ determined by $A$ given in Theorem 2.17. The proof is complete.

Now we prove that the assumptions of Theorem 2.21 concerning $D_{u, A, t}^{N}$ are satisfied.

TheOREM 2.22. Let $A, d(t)=\left(d_{1}(t), d_{2}(t), d_{12}(t), d_{4}(t)\right)$, and $\left(u^{N}, x^{1}, x^{2}\right)$ be as in Theorem 2.16. Let $D_{u, A, t}^{N}$ be defined by 2.21. Then for any $u_{N-3}=$ $u \in[0,1 / \sqrt{3})$ and $t \in \mathbb{R}$,

$$
\operatorname{det}\left(D_{u, A, t}^{N}\right)=\sum_{j=0}^{2(N-4)+4} c_{j, N}(u) t^{j},
$$

where the functions $c_{j, N}$ are continuous for $j=0, \ldots, 2(N-4)+4$ and

$$
c_{2 N-4, N}(u) \neq 0 \text {. }
$$

The same holds if we replace $A,\left(d(t), u^{N}, x^{1}, x^{2}\right)$ from Theorem 2.16 by those from Theorem 2.17 and assume that $u_{N-3}=u \in[1 / \sqrt{3}, 1)$.

Proof. First we assume that $N=4$. Let $g_{u_{1}, A, t}^{4}$ be as in Theorem 2.16 . We will differentiate the function $h_{u_{1}, A, t}^{4}$ given in Theorem 2.16 with respect to the following variables:

$$
\left(w_{1}, \ldots, w_{8}\right)=\left(x_{1}^{1}, x_{2}^{1}, x_{3}^{1}, x_{4}^{1}, b_{1}, b_{2}, b_{12}, b_{4}\right)
$$

and

$$
\left(w_{9}, \ldots, w_{14}\right)=\left(x_{1}^{2}, x_{2}^{2}, x_{3}^{2}, v_{2}, v_{3}, v_{4}\right)
$$


Set

$$
\begin{gathered}
X=\left(x_{1}, b, b, x_{4}, 0,-1 / \sqrt{2}, 1 / \sqrt{2}, 0\right), \\
B B=\left(b_{1}, b_{2}, 0, z\right) \quad \text { and } \quad v=\left(u_{1}, c, c, u_{4}\right) .
\end{gathered}
$$

By elementary but tedious calculations (see also the file theorem 2.22 a.nb at our web site) the $14 \times 14$ symmetric matrix $C=D_{u_{1}, A, t}^{4}(X, B B, v)$ is given by

$$
C=\left(\begin{array}{cc}
D_{1} & B \\
B^{T} & D_{2}
\end{array}\right)
$$

where

$$
D_{1}=\left(\begin{array}{rrrrrrr}
2\left(u_{1}^{2}-b_{1}\right) & 2 c u_{1} & 2 c u_{1} & -2 u_{1} u_{4} & -2 x_{1} & 0 & 0 \\
2 c u_{1} & 2\left(c^{2}-b_{1}\right) & -2 c^{2} & -2 c u_{4} & -2 b & 0 & 1 / \sqrt{2} \\
2 c u_{1} & -2 c^{2} & 2\left(c^{2}-b_{1}\right) & -2 c u_{4} & -2 b & 0 & -1 / \sqrt{2} \\
-2 u_{1} u_{4} & -2 c u_{4} & -2 c u_{4} & 2\left(u_{4}^{2}-b_{1}\right) & -2 x_{4} & 0 & 0 \\
-2 x_{1} & -2 b & -2 b & -2 x_{4} & 0 & 0 & 0 \\
0 & 0 & 0 & 0 & 0 & 0 & 0 \\
0 & 1 / \sqrt{2} & -1 / \sqrt{2} & 0 & 0 & 0 & 0
\end{array}\right),
$$

(2.25) $\quad D_{2}=$

$$
\left(\begin{array}{rrrrrrr}
0 & 0 & 0 & 0 & -2 c & -2 c & -2 u_{4} \\
0 & 2\left(u_{1}^{2}-b_{2}\right) & 2 c u_{1} & 2 c u_{1} & -\sqrt{2} u_{1} & \sqrt{2} u_{1} & 0 \\
0 & 2 c u_{1} & 2\left(c^{2}-b_{2}\right) & -2 c^{2} & -3 \sqrt{2} c & -\sqrt{2} c & 0 \\
0 & 2 c u_{1} & -2 c^{2} & 2\left(c^{2}-b_{2}\right) & \sqrt{2} c & 3 \sqrt{2} c & 0 \\
-2 c & -\sqrt{2} u_{1} & -3 \sqrt{2} c & \sqrt{2} c & 1+2\left(b^{2}-z\right) & 1-2 b^{2} & -2 b x_{4} \\
-2 c & \sqrt{2} u_{1} & -\sqrt{2} c & 3 \sqrt{2} c & 1-2 b^{2} & 1+2\left(b^{2}-z\right) & -2 b x_{4} \\
-2 u_{4} & 0 & 0 & 0 & -2 b x_{4} & -2 b x_{4} & 2\left(x_{4}^{2}-z\right)
\end{array}\right)
$$

and

(2.26) $\quad B^{T}=$

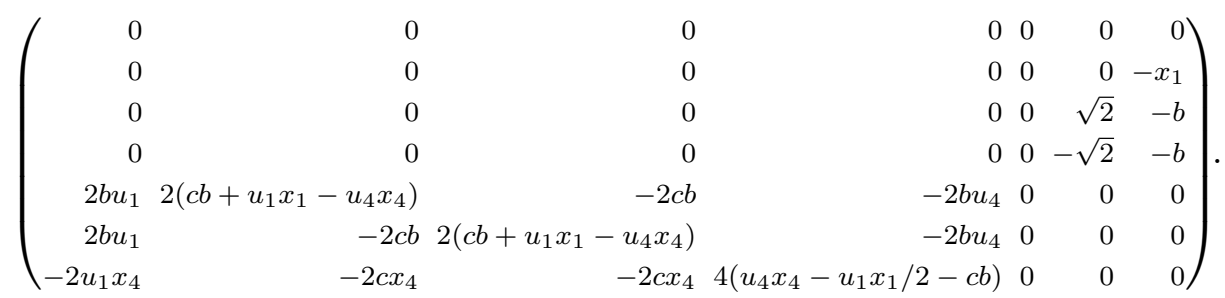

Notice that in the 6 th row of $C$ the only non-zero elements are $c_{6,10}=$ $-c_{6,11}=\sqrt{2}$ and in the 8 th row of $C$ the only elements which could be different from 0 are $c_{8,12}=c_{8,13}=-2 c$ and $c_{8,14}=-2 u_{4}$. Consequently, 
applying the symmetry of $C$, adding the 10 th row to the 11 th, the 10 th column to the 11 th column, subtracting the 14 th row multiplied by $c / u_{4}$ from the 12th and 13th rows, and subtracting the 14th column multiplied by $c / u_{4}$ from the 12 th and the 13 th columns, we obtain

$$
\operatorname{det}(C)=8 u_{4}^{2} \operatorname{det}(A),
$$

where $A$ is a $10 \times 10$ symmetric matrix of the form

$$
A=\left(\begin{array}{cc}
A_{1} & F \\
F^{T} & A_{2}
\end{array}\right)
$$

with

$$
A_{1}=\left(\begin{array}{rrrrrr}
2\left(u_{1}^{2}-b_{1}\right) & 2 c u_{1} & 2 c u_{1} & -2 u_{1} u_{4} & -2 x_{1} & 0 \\
2 c u_{1} & 2\left(c^{2}-b_{1}\right) & -2 c^{2} & -2 c u_{4} & -2 b & 1 / \sqrt{2} \\
2 c u_{1} & -2 c^{2} & 2\left(c^{2}-b_{1}\right) & -2 c u_{4} & -2 b & -1 / \sqrt{2} \\
-2 u_{1} u_{4} & -2 c u_{4} & -2 c u_{4} & 2\left(u_{4}^{2}-b_{1}\right) & -2 x_{4} & 0 \\
-2 x_{1} & -2 b & -2 b & -2 x_{4} & 0 & 0 \\
0 & 1 / \sqrt{2} & -1 / \sqrt{2} & 0 & 0 & 0
\end{array}\right) \text {, }
$$

$$
A_{2}=\left(\begin{array}{rrrr}
2\left(u_{1}^{2}-b_{2}\right) & 4 c u_{1} & -\sqrt{2} u_{1} & \sqrt{2} u_{1} \\
4 c u_{1} & -4 b_{2} & -2 \sqrt{2} c & 2 \sqrt{2} c \\
-\sqrt{2} u_{1} & -\sqrt{2} c & a_{3,3}-\left(2+2 c /\left(u_{4}\right)^{2}\right) z & a_{3,4}-2\left(c /\left(u_{4}\right)^{2}\right) z \\
\sqrt{2} u_{1} & \sqrt{2} c & a_{4,3}-2\left(c /\left(u_{4}\right)^{2}\right) z & a_{4,4}-\left(2+2 c /\left(u_{4}\right)^{2}\right) z
\end{array}\right),
$$

where $a_{3,4}=a_{4,3}$ and $a_{3,3}=a_{4,4}$ do not depend on $b_{2}$ and $z$. Also observe that the entries of $B^{T}$ do not depend on $b_{2}$ and $z$, hence the same is true for $F$. Now we calculate the coefficient $c_{4,4}\left(u_{1}\right)$ of $\operatorname{det}\left(D_{u_{1}, A, t}^{4}\right)$. To do this, we apply Lemmas 2.9 and 2.10 . Notice that

$$
\operatorname{det}(C(t))=\operatorname{det}\left(D_{u_{1}, A, t}^{4}(X, B B, v)\right)=8 u_{4}^{2} \operatorname{det}(A(t)),
$$

where $C(t)$ and $A(t)$ denote the above matrices $C$ and $A$ with $z$ replaced by $z+t /\left(2 u_{4}\right), b_{2}=b_{1}+t / \sqrt{2}$ and with $x_{1}=\sqrt{2} u_{1}, b=1 / \sqrt{6}, x_{4}=$ $-\sqrt{2\left(1-3 u_{1}^{2}\right)} / \sqrt{3}$. By Lemma 2.10 .

$$
c_{4,4}\left(u_{1}\right)=8 u_{4}^{2} \operatorname{det}\left(A_{1}\right) \operatorname{det}(E),
$$

where

$$
E=\left(\begin{array}{rrrr}
-\sqrt{2} & 0 & 0 & 0 \\
0 & -2 \sqrt{2} & 0 & 0 \\
0 & 0 & -\left(u_{4}^{2}+c\right) / u_{4}^{3} & -c / u_{4}^{3} \\
0 & 0 & -c / u_{4}^{3} & -\left(u_{4}^{2}+c\right) / u_{4}^{3}
\end{array}\right)
$$


Since $u_{1} \in[0,1 / \sqrt{3})$ and $u_{4}=\sqrt{1 / 3-u_{1}^{2}}>0, E$ is well-defined and $\operatorname{det}(E) \neq 0$. Notice that by Theorem 2.16 and Lemma 2.9 , $\operatorname{det}\left(A_{1}\right) \neq 0$. Consequently,

$$
c_{4,4}\left(u_{1}\right)=8 u_{4}^{2} \operatorname{det}\left(A_{1}\right) \operatorname{det}(E) \neq 0,
$$

which shows our claim.

Now assume that $N=4$ and let $A,\left(x^{1}, x^{2}, u^{4}, d\right)$ be as in Theorem 2.17 . In this case we have $u_{4}=0$ and $x_{4}^{1}=0$. Reasoning in a similar way (see also the file theorem $2.22 \mathrm{~b}$.nb at our web site) we get

$$
\operatorname{det}(C)=8\left(1-u_{1}^{2}\right) \operatorname{det}(A),
$$

where $A$ is a $10 \times 10$ symmetric matrix of the form

$$
A=\left(\begin{array}{cc}
A_{1} & F \\
F^{T} & A_{2}
\end{array}\right),
$$

where $A_{1}$ is as in the previous case and

$$
A_{2}=\left(\begin{array}{rrrr}
2\left(u_{1}^{2}-b_{2}\right) & 4 c u_{1} & \sqrt{2} u_{1} & 0 \\
4 c u_{1} & -4 b_{2} & 4 \sqrt{2} c & 0 \\
2 \sqrt{2} u_{1} & 4 \sqrt{2} c & d_{3,3}-4 z & 0 \\
0 & 0 & 0 & -2 z
\end{array}\right),
$$

Also, as in the previous case, the entries of $F$ do not depend on $z$ or $b_{2}$. Moreover, the entries of $A_{1}$ and $A_{2}$ do not depend on $a_{N, j}$ for $j=N-3$, $N-2, N-1$, which are not fixed, for $A$ given by (2.18), as they were in Theorem 2.16. Now we calculate the coefficient $c_{4,4}\left(u_{1}\right)$ of $\operatorname{det}\left(D_{u_{1}, A, t}^{4}\right)$. To do this, we apply Lemmas 2.9 and 2.10 . Notice that

$$
\operatorname{det}(C(t))=\operatorname{det}\left(D_{u_{1}, A, t}^{4}(X, B B, v)\right)=4\left(1-u_{1}^{2}\right) \operatorname{det}(A(t)),
$$

where $C(t)$ and $A(t)$ denote the above matrices $C$ and $A$ with $z$ replaced by $z+t /\left(2 u_{3}\right)$ and $b_{2}=b_{1}+t / \sqrt{2}$ and with $x_{1}=0, b=1 / \sqrt{2}, x_{4}=0$. By Lemma 2.10 ,

where

$$
c_{4,4}\left(u_{1}\right)=4\left(1-u_{1}^{2}\right) \operatorname{det}\left(A_{1}\right) \operatorname{det}(E),
$$

$$
E=\left(\begin{array}{rrrr}
-\sqrt{2} & 0 & 0 & 0 \\
0 & -2 \sqrt{2} & 0 & 0 \\
0 & 0 & -2 / u_{3} & 0 \\
0 & 0 & 0 & -4 / u_{3}
\end{array}\right) .
$$

Since $u_{1} \in[0,1)$ and $u_{3}=\sqrt{1-u_{1}^{2}}>0, E$ is well-defined and $\operatorname{det}(E) \neq 0$. Notice that by Theorem 2.17 and Lemma 2.9 , $\operatorname{det}\left(A_{1}\right) \neq 0$. Consequently,

$$
c_{4,4}\left(u_{1}\right)=4\left(1-u_{1}^{2}\right) \operatorname{det}\left(A_{1}\right) \operatorname{det}(E) \neq 0,
$$

which shows our claim. 
Now take any $N>4$. We show that the proof of this case practically reduces to the proof given for $N=4$. First assume that $A,\left(x^{1}, x^{2}, u^{N}, d(t)\right)$ are as in Theorem 2.16. We will differentiate with respect to the following variables:

$$
\begin{gathered}
\left(w_{1}, \ldots, w_{3(N-4)}\right)=\left(x_{1}^{1}, x_{1}^{2}, u_{1}, \ldots, x_{N-4}^{1}, x_{N-4}^{2}, u_{N-4}\right), \\
\left(w_{3(N-4)+1}, \ldots, w_{3 N+2}\right) \\
=\left(x_{N-3}^{1}, x_{N-2}^{1}, x_{N-1}^{1}, x_{N}^{1}, x_{N-3}^{2}, x_{N-2}^{2}, x_{N-1}^{2}, b_{1}, b_{2}, b_{12}, b_{4}, u_{N-2}, u_{N-1}, u_{N}\right) .
\end{gathered}
$$

(We do not differentiate with respect to $x_{N}^{2}$ and $u_{N-3}$.) Now we show that (since $u_{j}=x_{j}^{1}=x_{j}^{2}=0$ for $j=1, \ldots, N-4$ ) the matrix $C_{N}$ corresponding to our case has the form

$$
C_{N}=\left(\begin{array}{rrrrr}
W_{1} & 0 & \ldots & 0 & 0 \\
0 & W_{2} & \ldots & 0 & 0 \\
\ldots & \ldots & \ldots & \ldots & \ldots \\
0 & 0 & \ldots & W_{N-4} & 0 \\
0 & 0 & \ldots & 0 & C_{4}
\end{array}\right)
$$

where $C_{4}$ denotes the matrix obtained for

$$
\begin{aligned}
X & =\left(x_{N-3}^{1}, x_{N-2}^{1}, x_{N-1}^{1}, x_{N}^{1}, x_{N-3}^{2}, x_{N-2}^{2}, x_{N-1}^{2}, x_{N}^{2}\right), \\
u^{4} & =\left(u_{N-3}, u_{N-2}, u_{N-1}, u_{N}\right), \quad b=\left(d_{1}(t), d_{2}(t), d_{12}(t), d_{4}(t)\right)
\end{aligned}
$$

in the case $N=4$. Here, for $i=1, \ldots, N-4$,

$$
W_{i}=\left(\begin{array}{rrr}
-2 b_{1} & 0 & w_{i, 1} \\
0 & -2 b_{2} & w_{i, 2} \\
w_{i, 1} & w_{i, 2} & -2 z
\end{array}\right)
$$

where

$$
w_{i, k}=\sum_{j=N-3}^{N} a_{i j} u_{j} x_{j}^{k}
$$

for $k=1,2$. Indeed for any $j=1, \ldots, N$,

$$
\frac{\partial h_{u_{1}, A, t}^{N}}{\partial x_{j}^{1}}\left(x^{1}, x^{2}, u, d(t)\right)=2\left(\sum_{k=1}^{N} a_{j k} x_{k}^{1} u_{j} u_{k}-d_{12}(t) x_{j}^{2}-d_{1}(t) x_{j}^{1}\right)
$$

and

$$
\frac{\partial h_{u_{1}, A, t}^{N}}{\partial u_{j}}\left(x^{1}, x^{2}, u, d(t)\right)=2\left(\sum_{k=1}^{N} a_{j k} u_{k}\left\langle x_{j}, x_{k}\right\rangle_{2}-d_{4}(t) u_{j}\right) .
$$

Hence for any $j=1, \ldots, N-4$, 


$$
\frac{\partial^{2} h_{u, A, t}^{N}}{\partial x_{j}^{1} \partial w_{l}}\left(x^{1}, x^{2}, u, d(t)\right)=0
$$

for $w_{l} \neq x_{j}^{1}$ and $w_{l} \neq u_{j}$. The same reasoning applies if we differentiate with respect to $x_{j}^{2}, j=1, \ldots, N-4$. Analogously, for $j=1, \ldots, N-4$,

$$
\frac{\partial^{2} h_{u_{1}, A, t}^{N}}{\partial u_{j} \partial w_{l}}\left(x^{1}, x^{2}, u, d(t)\right)=0
$$

for $w_{l} \neq x_{j}^{i}, i=1,2$ and $w_{l} \neq u_{j}$. Also for

$$
\begin{aligned}
w_{k}, w_{j} \in\left\{x_{N-3}^{1}, x_{N-2}^{1}, x_{N-1}^{1}, x_{N}^{1}, x_{N-3}^{2}, x_{N-2}^{2}, x_{N-1}^{2}, u_{N-2}, u_{N-1}, u_{N}\right\} \\
\cup\left\{b_{1}, b_{2}, b_{12}, b_{4}\right\}
\end{aligned}
$$

we have

$$
\frac{\partial^{2} h_{u_{1}, A, t}^{N}}{\partial w_{j} \partial w_{k}}\left(x^{1}, x^{2}, u^{N}, d\right)=\frac{\partial^{2} h_{u_{1}, A, t}^{4}}{\partial w_{j} \partial w_{k}}\left(z^{1}, z^{2}, v, d\right),
$$

where $h_{u_{1}, A, t}^{4}$ is the function from Theorem 2.16 corresponding to $N=4$ and

$$
\begin{aligned}
& z^{1}=\left(x_{N-3}^{1}, x_{N-2}^{1}, x_{N-1}^{1}, x_{N}^{1}\right), \\
& z^{2}=\left(x_{N-3}^{2}, x_{N-2}^{2}, x_{N-1}^{2}\right), \quad v=\left(u_{N-2}, u_{N-1}, u_{N}\right) .
\end{aligned}
$$

This shows our claim concerning the matrix $C_{N}$.

Since $w_{i, k}$ for $k=1,2$ and $i=1, \ldots, N-4$ do not depend on $b_{2}$ and in our situation $b_{1}=2 / 3, b_{2}=2 / 3+t / \sqrt{2}, z=4 / 3+t /\left(2 u_{n}\right)$ by the proof given in the case $N=4$,

$$
c_{4+2(N-4), N}\left(u_{N-3}\right) \neq 0
$$

for any $u_{N-3} \in[0,1 / \sqrt{3})$, which completes the proof for $N>4$ in the case of $A$ from Theorem 2.16. The case of $A$ from Theorem 2.17 and $N>4$ can be proved in exactly the same way, so we omit it.

3. A proof of the Grünbaum conjecture. Our proof of the Grünbaum conjecture uses an induction argument. Notice that by Lemma 2.5 we have $\lambda_{2}^{3}=4 / 3$. First we show that $\lambda_{2}^{4}=4 / 3$. Then assuming $\lambda_{2}^{N}=4 / 3$ we demonstrate that $\lambda_{2}^{N+1}=4 / 3$.

Theorem 3.1. Fix $N \in \mathbb{N}$ with $N \geq 4$ and $u_{N-3} \in[0,1]$. Let

$$
f_{u_{N-3}}^{N}\left(u_{1}, \ldots, u_{N-4}, u_{N-2}, u_{N-1}, u_{N}, x^{1}, x^{2}\right)=\sum_{i, j=1}^{N} u_{i} u_{j}\left|\left\langle x_{i}, x_{j}\right\rangle_{2}\right| .
$$

Let $M_{u, N}=\max f_{u}^{N}$ under constraints 2.2 and 2.3). Then for any $u_{N-3} \in$ $[0,1 / \sqrt{3})$,

$$
M_{u_{N-3}, N}=4 / 3
$$


and for any $u_{N-3} \in[1 / \sqrt{3}, 1]$,

$$
M_{u_{N-3}, N}=1+\left(\sqrt{4 u_{N-3}^{2}-3 u_{N-3}^{4}}-u_{N-3}^{2}\right) / 2 .
$$

Proof. We proceed by induction on $N$. First assume $N=4$. Define

$$
U_{4}=\left\{u_{1} \in[0,1 / \sqrt{3}): M_{u_{1}, 4}=4 / 3\right\} .
$$

By Lemmas 2.5 and 2.6, $0 \in U_{4}$. Now we show that $U_{4}$ is an open set. Fix $u_{1} \in U$. First we consider the case $u_{1}=0$. We apply Theorems 2.21 and 2.22. Assume that there exist $\left\{w_{n}\right\} \subset \mathbb{R}_{+}$with $w_{n} \rightarrow 0$ and $w_{n} \notin U$ for any $n \in \mathbb{N}$. Let $\left(Z_{w_{n}}, M_{w_{n}}(t)\right)$ be as in the proof of Theorems 2.21. Passing to a convergent subsequence if necessary, and reasoning as in Theorem 2.21, we can assume that $\left(Z_{w_{n}}, M_{w_{n}}(t)\right) \rightarrow\left(X_{0}, L_{0}\right)$. Let $Z_{w_{n}}=\left(w_{n}^{4}, z_{1 n}, z_{2 n}\right)$. Since $Z_{w_{n}} \rightarrow X_{0}$ we have

$$
\operatorname{sgn}\left\langle z_{i n}, z_{j n}\right\rangle_{2}=a_{i j}
$$

for $i, j=2,3,4$ and $n \geq n_{0}$, where the matrix $a_{i j}$ is given by (2.18) for $N=4$. Without loss of generality, passing to a convergent subsequence if necessary we can assume that for $n \geq n_{0}$,

$$
\operatorname{sgn}\left\langle z_{1 n}, z_{j n}\right\rangle_{2}=z_{j}
$$

for $j=2,3,4$, where $z_{j}= \pm 1$. By Lemma 2.7 we have to consider two cases:

(a) $z_{2}=z_{3}=z_{4}=1$;

(b) $z_{2}=z_{3}=1, z_{4}=-1$.

If (a) holds true, then by Theorems 2.21, 2.18 (applied to $u_{N-3}=0$ ) and 2.22 we get

$$
M_{w_{n}, 4}=4\left(1-w_{n}^{2}\right) / 3<2 / 3+2 / 3=4 / 3
$$

for $n \geq n_{0}$, which by Theorem 2.16 leads to a contradiction. (Since $u_{1}=0$, $D_{u_{1}, A, t}^{4}$ is the same for $h_{u_{1}, A, t}^{4}$ from Theorem 2.18 as for $h_{u_{1}, A, t}$ from Theorem 2.16). If (b) holds true, by Theorems 2.11, 2.22 and 2.16 we get a contradiction with Theorem 2.21. Consequently, there exists an interval $[0, v) \subset U_{4}$.

Now assume that $v=u_{1} \in U$ and $v>0$. Assume $w_{n} \rightarrow v$ and $w_{n} \notin U_{4}$ for $n \in \mathbb{N}$. Let $\left(Z_{u_{n}}, M_{u_{n}}\right)$ be as in Theorem 2.21. Without loss of generality we can assume that $\left(Z_{w_{n}}, M_{w_{n}}(t)\right) \rightarrow\left(X_{v}, L_{v}(t)\right)$. Let $Z_{w_{n}}=\left(w_{n}^{4}, z_{1 n}, z_{2 n}\right)$. Since $Z_{w_{n}} \rightarrow X_{v}$ we have

$$
\operatorname{sgn}\left\langle z_{i n}, z_{j n}\right\rangle_{2}=a_{i j}
$$

for $i, j=1,2,3,4$ for $n \geq n_{0}$, where the matrix $\left(a_{i j}\right)$ is as in Theorem 2.16 for $N=4$. Applying Theorem 2.21, we get $w_{n} \in U$ for $n \geq n_{0}$, a contradiction. Hence the set $U_{4}$ is open.

It is easy to see that $U_{4}$ is also closed. Since $0 \in U_{4}$ and $[0,1 / \sqrt{3})$ is connected, $U_{4}=[0,1 / \sqrt{3})$. Observe that by the continuity of the function 
$u_{N-3} \mapsto f_{u_{N-3}}^{N}$, we get

$$
M_{1 / \sqrt{3}, 4}=4 / 3 .
$$

Now define

$$
W_{4}=\left\{u_{1} \in[1 / \sqrt{3}, 1): M_{u_{1}, 4}=1+\left(\sqrt{4 u_{1}^{2}-3 u_{1}^{4}}-u_{1}^{2}\right) / 2\right\} .
$$

By the above reasoning $1 / \sqrt{3} \in W_{4}$. Let $v=u_{1} \in W_{4}$. Assume that $w_{n} \rightarrow v$ and $w_{n} \notin W_{4}$. Applying Theorem 2.17 and proceeding as above we find that $\left(Z_{w_{n}}, M_{w_{n}}(t)\right) \rightarrow\left(X_{v}, L_{v}(t)\right)$. Also reasoning as above, passing to a convergent subsequence if necessary, we can assume that

$$
f_{w_{n}}^{4}=f_{w_{n}, A}^{4},
$$

where $A$ is a fixed matrix satisfying (2.18). By Theorems 2.17, 2.21 and 2.22 $w_{n} \in W_{4}$ for $n \geq n_{0}$, a contradiction. Hence $W_{4}$ is an open set. Reasoning as above we get

$$
W_{4}=[1 / \sqrt{3}, 1),
$$

which completes the proof for $N=4$. (It is easy to see that $M_{1,4}=1$.)

Now assume that our formula for $M_{u_{N-3}, N}$ holds true. We will show that it holds for $M_{u_{N+1-3}, N+1}$. We will proceed in the same way as in the case $N=4$. Define

$$
U_{N+1}=\left\{u_{N-2} \in[0,1 / \sqrt{3}): M_{u_{N-2}, N+1}=4 / 3\right\} .
$$

By the induction hypothesis and Lemma 2.6, $0 \in U_{N+1}$. Reasoning as in the $N=4$ case and applying Theorems 2.16, 2.18, 2.21 and 2.22, we show that $U_{N+1}$ is an open set. It is clear that it is closed. Hence $U_{N+1}=[0,1 / \sqrt{3})$. Again by the continuity of $u_{N+1-3} \mapsto f_{u_{N+1-3}}^{N+1}$ we get

$$
M_{1 / \sqrt{3}, N+1}=4 / 3 \text {. }
$$

Define

$W_{N+1}$

$$
=\left\{u_{N-2} \in[1 / \sqrt{3}, 1): M_{u_{N-2}, N+1}=1+\left(\sqrt{4 u_{N-2}^{2}-3 u_{N-2}^{4}}-u_{N-2}^{2}\right) / 2\right\} .
$$

By the above reasoning $1 / \sqrt{3} \in W_{N+1}$. Applying Theorems 2.17, 2.21 and 2.22 and proceeding as in the case $N=4$, we get

$$
W_{N+1}=[1 / \sqrt{3}, 1) .
$$

It is easy to see that $M_{1, N+1}=1$. The proof is complete.

THEOREM 3.2 .

$$
\lambda_{2}=4 / 3 .
$$

Proof. By Theorems 3.1, 2.4 and Lemma 2.13 ,

$$
\lambda_{2}^{N}=4 / 3
$$


for any $N \in \mathbb{N}$ with $N \geq 3$. Let $V \subset l_{\infty}$ be so chosen that $\operatorname{dim}(V)=2$ and $\lambda_{2}=\lambda(V)$. For any $\epsilon>0$ we can find $N \in \mathbb{N}$ and $V_{N} \subset l_{\infty}^{(N)}$ such that

$$
\ln \left(d\left(V_{N}, V\right)\right) \leq \epsilon,
$$

where $d$ denotes the Banach-Mazur distance. Since

$$
\left|\ln \left(\lambda\left(V_{N}\right)\right)-\ln (\lambda(V))\right| \leq \ln \left(d\left(V_{N}, V\right)\right)
$$

(see e.g. [13, p. 113]), we obtain

$$
\lambda_{2}=\lambda(V) \leq \lambda\left(V_{N}\right) e^{\epsilon} \leq \lambda_{2}^{N} e^{\epsilon} .
$$

Consequently,

$$
\lim _{N} \lambda_{2}^{N}=\lambda_{2},
$$

which shows that $\lambda_{2}=4 / 3$. The proof is complete.

REMARK 3.3. Notice that in [4, it has been proven that

$$
\lambda(V) \leq 4 / 3
$$

for any two-dimensional, real, unconditional Banach space. Recall that a two-dimensional, real Banach space $V$ is called unconditional if there exists a basis $v^{1}, v^{2}$ of $V$ such that for any $a_{1}, a_{2} \in \mathbb{R}$ and $\epsilon_{1}, \epsilon_{2} \in\{-1,1\}$,

$$
\left\|a_{1} v^{1}+a_{2} v^{2}\right\|=\left\|\epsilon_{1} a_{1} v^{1}+\epsilon_{2} a_{2} v^{2}\right\| .
$$

Acknowledgements. The authors are indebted to the referee for his valuable remarks concerning the final version of this paper.

\section{References}

[1] B. L. Chalmers and G. Lewicki, Symmetric subspaces of $l_{1}$ with large projection constants, Studia Math. 134 (1999), 119-133.

[2] —, 一, Symmetric spaces with maximal projection constants, J. Funct. Anal. 200 (2003), 1-22.

[3] - - - Three-dimensional subspace of $l_{\infty}^{(5)}$ with maximal projection constant, ibid. 257 (2009), 553-592.

[4] B. L. Chalmers and B. Shekhtman, Extension constants of unconditional two-dimensional operators, Linear Algebra Appl. 240 (1996), 173-182.

[5] B. Grünbaum, Projection constants, Trans. Amer. Math. Soc. 95 (1960), 451-465.

[6] M. I. Kadets and M. G. Snobar, Certain functionals on the Minkowski compactum, Math. Notes 10 (1971), 694-696.

[7] H. König, Spaces with large projection constants, Israel J. Math. 50 (1985), 181-188.

[8] H. König, D. R. Lewis and P. K. Lin, Finite-dimensional projection constants, Studia Math. 75 (1983), 341-358.

[9] H. König and N. Tomczak-Jaegermann, Bounds for projection constants and 1summing norms, Trans. Amer. Math. Soc. 320 (1990), 799-823.

[10] —, 一, Norms of minimal projections, J. Funct. Anal. 119 (1994), 253-280. 
[11] H. König, C. Schütt and N. Tomczak-Jaegermann, Projection constants of symmetric spaces and variants of Khintchine's inequality, J. Reine Angew. Math. 511 (1999), 1-42.

[12] D. Rutovitz, Some parameters associated with finite-dimensional Banach spaces, J. London Math. Soc. 40 (1965), 241-255.

[13] P. Wojtaszczyk, Banach Spaces for Analysts, Cambridge Univ. Press, Cambridge, 1991.

Bruce L. Chalmers

Department of Mathematics

University of California

Riverside, CA 92521, U.S.A.

E-mail: blc@math.ucr.edu
Grzegorz Lewicki Institute of Mathematics Jagiellonian University 30-348 Kraków, Poland E-mail: Grzegorz.Lewicki@im.uj.edu.pl

Received December 22, 2008

Revised version July 5, 2010 